\title{
A Prospective Review: The Significance of Procalcitonin Levels in the Evaluation of Pediatric Patients Presenting to the Emergency Department with Fever (Pedi-SPEED I)
}

\author{
Candi Sturges ${ }^{1}$, Chanin Wright ${ }^{1,2,3}$ Dominic Lucia $^{1,4}$ and Amanda Farris ${ }^{1,2}$ \\ 1. McLane Children's Hospital-Baylor Scott \& White Healthcare, Temple, TX, USA \\ 2. Department of Pediatrics, Texas A\&M Health Science Center College of Medicine, Temple, TX, USA \\ 3. College of Pharmacy, University of Texas, Temple, TX, USA \\ 4. Department of Emergency Medicine, Texas A\&M Health Science Center College of Medicine, Temple, TX, USA
}

\begin{abstract}
Purpose: This study was formulated to educate physicians and medical residents on procalcitonin (PCT). The need for this study is based on lack of information and studies in the United States in the pediatric population regarding PCT, and also due to the lack of awareness by the Baylor Scott \& White McLane Children's residents in the implications and benefits of PCT. There are several instances where it can be valuable in the diagnosis and treatment of bacterial infections, which in turn, can decrease patient's length of stay, hospital costs, and patient exposure to unnecessary antibiotics, without increasing morbidity or mortality. Methods: An educational, qualitative analysis of PCT awareness is assessed utilizing pre and post surveys. These surveys will be given to pediatric and emergency medicine residents on paper before and after an educational presentation about PCT and its importance as a detection method for infection. Surveys consist of six closed-ended questions on the pre-survey and eight open and close-ended questions on the post-survey about PCT, how it works, general use, and how it can be beneficial. Results: Pediatric and emergency medicine residents were asked to participate in this study at a random monthly department meeting, selected by primary investigator and department head. Residents were asked to complete the pre-survey, listened to a short presentation, and completed the post survey. Comparing the pre-survey to post-survey, there was an overall improvement for both groups for questions 1-5 which showed statistical significance $(P<0.05)$. No statistical difference was found between groups comparing emergency medicine vs. pediatric departments, interns vs. residents and each subgroup pre-survey vs. post-survey for questions 6 and 7. The open-ended post-survey question identified factors for obtaining PCT levels with attending physician's preference as the most common response. Conclusions: This prospective study identified gaps in PCT knowledge base, an overall increase in PCT comprehension by pediatric and emergency medicine residents, and identified factors for obtaining PCT levels upon patient admission.
\end{abstract}

Key words: Procalcitonin, PCT, pediatric, infectious disease, emergency medicine.

\section{Introduction}

In the past few decades, there have been several diagnostic methods proposed as an adjunct to clinical judgment to aid in the identification of bacterial illnesses which present with fever, along with no clear source of infection. Biomarkers are just one of the many methods proposed to help clinicians in diagnosing these types of patients. These earlier biomarkers have included WBC (white blood cell

Corresponding author: Candi Sturges, Pharm.D., research field: pediatric emergency medicine. E-mail: csturges@sw.org. count), ANC (absolute neutrophil count), and CRP (C-reactive protein). However, PCT (procalcitonin) is a new biomarker that has been used in adult and pediatric patients to help detect systemic infections, and has been shown in studies to be more specific for bacterial than viral infections [1].

Procalcitonin is a precursor to the parathyroid hormone calcitonin, which has been shown to have a rapid, increased response to infection and inflammatory stimuli. Levels of PCT are almost undetectable during normal physiological conditions 

Presenting to the Emergency Department with Fever (Pedi-SPEED I)

and can be elevated due to infectious conditions such as bronchitis, meningitis, pneumonia and other lower respiratory tract infections, pyelonephritis and urinary tract infections, sepsis or septic shock, and other conditions such as surgery/trauma, burns or cancer-related causes [1-4]. However, increased levels of PCT have been found within 2-4 hours of symptoms, and can peak between 6-24 hours, whereas CRP slowly elevates in 12 hours and peaks at 48-72 hours post-infectious stimuli $[1,2,5,6]$.

This study was formulated to educate medical residents on procalcitonin, based on the lack of information and studies in the United States in the pediatric population regarding PCT, and also due to the lack of awareness in the Baylor Scott \& White McLane Children's medical and emergency medicine residents in the implications and benefits of PCT. There are several instances where it can be valuable in the diagnosis and treatment of bacterial infections, which in turn, can decrease patient's length of stay, hospital costs, and patient exposure to unnecessary antibiotics, without increasing morbidity or mortality [7]. Pediatric and emergency medicine residents were targeted for this study as they are more likely to interact with this subset of patients and will be ordering the labs and diagnostics. The primary objective of Part $\mathrm{I}$ is to identify an increase in overall awareness in how procalcitonin can be used and its benefits, with a primary endpoint of an increase in the percentage of correct answers from pre-survey to post-survey. The secondary objective is to identify factors that contribute to residents ordering or not ordering a PCT level.

\section{Methods}

\subsection{Study Design}

An educational, qualitative analysis of PCT awareness will be assessed utilizing pre and post surveys. These surveys will be given to pediatric and emergency medicine residents on paper before and after an educational presentation about PCT and its importance as a detection method for infection. Surveys consist of six closed-ended questions in the pre-survey and seven close-ended questions in the post-survey about PCT, how it works, what it is used for, and how it can be beneficial (Appendix A). Results from the post-survey will be compared with the pre-survey results to identify improvement and learn understanding. The expected outcome is to see an increase in the percentage of correct answers and also see an increase in overall awareness in how PCT can be used and its benefits. All information will be de-identified by a randomly assigned number and information kept in a secure computer database.

\subsection{Subject Selection}

Pediatric and emergency medicine residents will be invited to participate in one of their monthly resident meetings. There are 41 emergency medicine residents and 22 pediatric residents. A sample size of 34 achieves $81 \%$ power to detect a mean of paired difference in percentage of correct answers of 10 with an estimated standard deviation of differences of 20 and with a statistically significant level of 0.05 determined using a two-sided paired t-test.

\subsection{Procedures/Methods}

Residents will be notified of the monthly resident meeting by their residency program director and will be asked for their participation in the study upon arrival to the selected meeting. Meetings are held separately from the other department and will only have one date and time for residents to participate in this study. Pre-surveys will be handed out and residents will be asked to complete the pre-survey prior to the educational presentation. They will be instructed to fill out the top section and answer each question to the best of their ability. After all pre-surveys are complete, they will be collected and logged by resident department type. Educational presentation will be given and questions will be answered appropriately. At the end of the presentation, the post-survey will be distributed and 


\section{A Prospective Review: The Significance of Procalcitonin Levels in the Evaluation of Pediatric Patients Presenting to the Emergency Department with Fever (Pedi-SPEED I)}

the residents will be asked to complete the post-survey to the best of their ability along with also completing the top section. Once all the post-surveys are completed they will be collected and logged by resident department type. Each survey will be graded for questions 1 through 6 and overall grade will be entered into an Excel spreadsheet to determine improvement. Responses on questions 7 and 8 will be used to determine overall favorability of procalcitonin.

\subsection{Data Analysis}

Survey of participant characteristics and survey results will be summarized using descriptive statistics: mean (standard deviation) for continuous variables and frequency (percent) for categorical variables. Percentage of correct answers will be compared between pre-survey and post-survey by paired t-test or Wilcoxon signed rank test. Favorability of PCT will be compared between pre-survey and post-survey by utilizing Wilcoxon signed rank test. A p-value of less than 0.05 will indicate a statistical significance. SAS 9.2 (SAS Institute INC, Cary, NC) will be used for data analysis.

\section{Results}

Pediatric and emergency medicine residents were asked to participate in this survey at a random monthly department meeting, selected by primary investigator and department head. Residents from both departments were $N=28$. Comparing the pre-survey to post-survey, there was an overall improvement for both groups for questions 1-5 which showed statistical significance (Table 1). There was no statistical difference between groups when comparing emergency medicine vs. pediatric departments, interns vs. residents and each subgroup pre-survey vs. post-survey for questions 6 and 7. An increased number of responses to question 7 for "Sometimes" identified, as this post-survey option stated PCT was to be used for specific patients in specific situations, which was the most promising answer to identify if residents learned the purpose for PCT and patients who showed the most benefit (Fig. 1). The final question on the post survey identified factors for obtaining PCT levels with Attending Physician's preference as the most common response. Other responses included the severity of patient's illness, cost of running PCT levels vs. CRP, time for lab turn-around, amount of blood loss due to testing purposes, and the overall need more research and information.

\section{Conclusion}

This prospective study identified gaps in PCT knowledge base, an overall increase in PCT comprehension by pediatric and emergency medicine residents, and identified factors for obtaining PCT levels at patient admission.

Table 1 Pre and post survey scoring.

\begin{tabular}{|c|c|c|c|c|}
\hline Variable & $\begin{array}{l}\text { Pre survey } \\
(N=28)\end{array}$ & $\begin{array}{l}\text { Post survey } \\
(N=28)\end{array}$ & $\begin{array}{l}\text { Difference of pre and } \\
\text { post survey results }\end{array}$ & $P$-value \\
\hline \multicolumn{5}{|c|}{ Q1: What is Procalcitonin (PCT)? } \\
\hline Correct Answer (\%) & $22(78.6 \%)$ & $28(100.0 \%)$ & $6(21.4 \%)$ & $0.0313 *$ \\
\hline \multicolumn{5}{|c|}{ Q2: How does it function in the body? } \\
\hline Correct Answer (\%) & $26(92.9 \%)$ & $28(100.0 \%)$ & $2(7.1 \%)$ & 0.5000 \\
\hline \multicolumn{5}{|c|}{ Q3: What can PCT be used for? } \\
\hline Correct Answer (\%) & $26(92.9 \%)$ & $28(100.0 \%)$ & $2(7.1 \%)$ & 0.5000 \\
\hline \multicolumn{5}{|c|}{ Q4: In what type of patients would knowing a PCT level be useful? } \\
\hline Correct Answer (\%) & $19(67.9 \%)$ & $28(100.0 \%)$ & $9(32.1 \%)$ & $0.0039 *$ \\
\hline \multicolumn{5}{|c|}{ Q5: How can the rapid PCT level results help in the treatment of critically ill patients? } \\
\hline Correct Answer (\%) & $28(100.0 \%)$ & $28(100.0 \%)$ & $0(0.0 \%)$ & N/A \\
\hline Results & $24.2(86.4 \%)$ & $28(100.0 \%)$ & $3.8(13.6 \%)$ & $0.0005^{*}$ \\
\hline
\end{tabular}

* Denotes statistically significant. 

Presenting to the Emergency Department with Fever (Pedi-SPEED I)

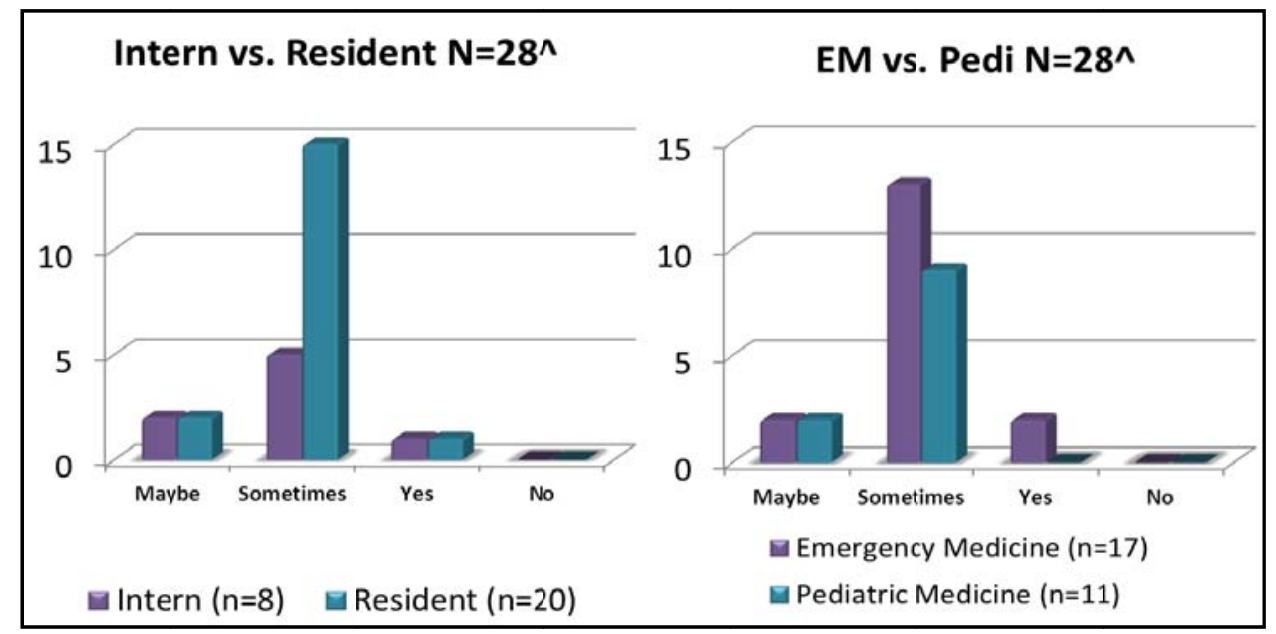

Fig. 1 Question 7 responses

\section{References}

[1] Schuetz, P., Albrich, W., and Mueller, B. 2011. "Procalcitonin for Diagnosis of Infection and Guide to Antibiotic Decisions: Past, Present and Future.” BMC Medicine. 9: 107. Accessed September 10, 2013. http://www.biomedcentral.com/1741-7015/9/107.

[2] Irwin, A., and Carrol, E. 2011. "Procalcitonin.” Arch. Dis. Child Educ. Prac.t Ed. 96: 228-33. Accessed August 24, 2013.

[3] Wacker, C., Prkno, A., Brunkhorst F. M., and Schlattmann, P. 2013. "Procalcitonin as a Diagnostic Marker for Sepsis: a Systematic Review and Meta-Analysis." Lancet Infect Dis. 13: 426-35. Accessed August 24, 2013. http://dx.doi.org/10.1016/S1473-3099(12)70323-7.

[4] Prasad, R., Kapoor, R., Mishra, O. P., Srivastava, R., and Kant Singh, U. 2013. "Serum Procalcitonin in Septic Meningitis.” Indian J. Pediatr. 80 (5): 365-70. Accessed August 24, 2013. http://link.springer.com/.

[5] Chiesa, C., Panero, A., Rossi, N., Stegagno, M., De Giusti,
M., Osborn, J. F., and Pacifico, L. 1998. "Reliability of Procalcitonin Concentrations for the Diagnosis of Sepsis in Critically Ill Neonates.” Clinical Infectious Diseases. 26: 664-72. Accessed August 24, 2013. http://cid.oxfordjournals.org/.

[6] Yo, C., Hsieh, P., Lee, S., Wu, J., Chang, S., Tasi, K., and Lee, C. 2012. "Comparison of the Test Characteristics of Procalcitonin to C-Reactive Protein and Leukocytosis for the Detection of Serious Bacterial Infections in Children Present with Fever without Source: A Systemic Review and Meta-analysis.” Annals of Emergency Medicine. 60 (5): 591-600. Accessed September 10, 2013. http://www.annemergmed.com/article/S0196-0644(12)00 520-3/abstract.

[7] Soni, N. J., Samson D. J., Galaydick, J. L., Vats, V., Huang, E. S., Aronson, N., and Pitrak, D. L. 2013. "Procalcitonin-Guided Antibiotic Therapy: A Systematic Review and Meta-analysis.” Journal of Hospital Medicine. 8 (9): 530-40. Accessed August 23, 2013. http://onlinelibrary.wiley.com/doi/10.1002/jhm.2067/abst ract. 
A Prospective Review: The Significance of Procalcitonin Levels in the Evaluation of Pediatric Patients Presenting to the Emergency Department with Fever (Pedi-SPEED I)

Appendix: Pre and Post Survey

Disclaimer: Completing this survey that states you are agreeing to participate in this research project as participation in the study is voluntary. Refusal to participate involves no penalty to the residents and will not affect their evaluations.
Specialty: $\square$ Emergency Medicine
CurrentTraining Level: $\square$ Intern
$\square$ Pediatric Medicine
$\square$ Resident

Last 4 digits of your pager \#:

\section{Procalcitonin Pre-Survey 2013}

1) What is Procalcitonin (PCT)?
a. Immunomodulator
b. Cytokine
c. Inflammatory biomarker
d. Monoclonal antibody

2) How does it function in the body?

a. PCT levels increase due to an inflammatory process ie: infection

b. PCT levels decrease amount of naturally occurring clotting factors

c. PCT levels increase in response to tissue damage

d. PCT levels increase to indicate calcium imbalances

3) What can PCT be used for?
a. To help guide antibiotic therapy
b. Decrease length of hospital stay
c. Method of possible antibiotic stewardship
d. All of the above

4) In what type of patients would knowing a PCT level be useful?
a. Patients presenting with broken bones or fractures
b. Patients presenting with a fever of unknown source
c. Patients $\leq 72$ hours of age presenting with cough and wheezing
d. Chronic disease patients presenting with exacerbations ie: cystic fibrosis

5) How can the rapid PCT level results help in the treatment of critically ill patients?
a. Indicate to start antibiotic therapies
b. Indicate to start antiviral therapies
c. Indicate to start respiratory therapies
d. Indicate to start anticoagulation therapies

6) Would knowing a patient's procalcitonin level affect your clinical practice?
a. Yes
b. No
c. Maybe
d. Unsure 

Presenting to the Emergency Department with Fever (Pedi-SPEED I)

Disclaimer: Completing this survey that states you are agreeing to participate in this research project as participation in the study is voluntary. Refusal to participate involves no penalty to the residents and will not affect their evaluations.
Speceialty: $\square$ Emerägency Mèdiciné
Current Training Level: $\square$ Intêrn
- Pediatric Medicine
$\square$ Resident

Last 4 digits of your pager $\#$ :

\section{Procalcitonin Post-Survey 2013}

1) What is Procalcitonin (PCT)?
a. Immunomodulator
b. Cytokine
c. Inflammatory biomarker
d. Monoclonal antibody

2) How does it function in the body?
a. PCT levels increase due to an inflammatory process ie: infection
b. PCT levels decrease amount of naturally occurring clotting factors
c. PCT levels increase in response to tissue damage
d. PCT levels increase to indicate calcium imbalances

3) What can PCT be used for?
a. To help guide antibiotic therapy
b. Decrease length of hospital stay
c. Method of possible antibiotic stewardship
d. All of the above

4) In what type of patients would knowing a PCT level be useful?
a. Patients presenting with broken bones or fractures
b. Patients presenting with a fever of unknown source
c. Patients $\leq 72$ hours of age presenting with cough and wheezing
d. Chronic disease patients presenting with exacerbations ie: cystic fibrosis

5) How can the rapid PCT level results help in the treatment of critically ill patients?
a. Indicate to start antibiotic therapies
b. Indicate to start antiviral therapies
c. Indicate to start respiratory therapies
d. Indicate to start anticoagulation therapies

6) Would knowing a patient's procalcitonin level affect yourclinical practice?
a. Yes
b. No
c. Maybe
d. Unsure

7) Do you plan to use procalcitonin levels as an important indicator in the near future?

a. Yes, every time a patient presents with a fever of unknown origin, sepsis or possible pneumonia, etc

b. Sometimes, I think this measurement would prove useful in specific patients and situations

c. No, I never plan to order PCT levels in the future as the current standard of care works

d. Maybe

8) What factors would influence your decision to order PCT levels? 